\section{Comment on "Measurement of the Intrinsic Dissipation of a Macroscopic System in the Quantum Regime"}

Recently, Cosmelli et al. (hereafter the authors) [1] measured the escape rate $(\Gamma)$ vs normalized flux bias $(X \equiv$ $\left.\Phi_{x} / \Phi_{0}\right)$ of an rf SQUID from a metastable fluxoid state at a temperature below $50 \mathrm{mK}$. The data were compared to calculations from which an effective damping resistance $R=4 \mathrm{M} \Omega$ was extracted. However, in the following discussion we show that, in Ref. [1], (i) the energy level structure used to calculate the escape rate was significantly incorrect, and (ii) treating system temperature as a free fitting parameter could not be justified. Therefore, the value of $R$ inferred from the data is unreliable.

In Ref. [1], the measured escape rate $\Gamma(X)$ was compared to the solution of the master equation. Using the SQUID parameters given in Ref. [1], we found the number of levels in the well is $N \simeq 11$ to 13 ; that is in a stark contrast to the value of 20 to 30 estimated by the authors. Furthermore, we found the parameter $\eta$, which is completely set by $Z_{0} \equiv \sqrt{L / C}, \beta_{L} \equiv 2 \pi L I_{c} / \Phi_{0}$, and $X$, varies smoothly from 660 to 700 for $-0.505<X<$ -0.485 . Thus, the value $\eta \approx 900$ obtained by the authors is $\sim 30 \%(\sim 6 \sigma)$ greater than the independently determined value. The calculated barrier height $\Delta U$, small oscillation frequency $\omega_{0}, \Delta U / \hbar \omega_{0}$, and $\eta \equiv 2 \pi d\left(\Delta U / \hbar \omega_{0}\right) / d X$ vs $X$ are shown in Fig. 1. Obviously, the result of rate calculation depends crucially on the level structure. Hence, the use of incorrect values of $N$ and $\eta$ is sufficient to raise question about the validity of the $\Gamma(X)$ calculations in Ref. [1].

The observed oscillations in $\Gamma(X)$ have been attributed to a depletion of the highest active level, denoted as the $n$th excited level with energy $E_{n}$, that contributes the most to escape. Roughly speaking, the amplitude of oscillations in $\Gamma(X)$ can be taken as a measure of how fast the level $n$ is being repopulated from below. Since the $n$th level couples most strongly to its nearest neighbors, a good approximation on the rate of repopulating it is given by

$$
\begin{aligned}
W_{n-1, n} & =W_{n, n-1} \exp \left[\left(E_{n-1}-E_{n}\right) / k_{B} T\right] \\
& \approx n(R C)^{-1} \exp \left(-\hbar \omega / k_{B} T\right),
\end{aligned}
$$

where $W_{n-1, n}$ is the transition rate from the $(n-1)$ th to the $n$th level, and $\omega$ is the level spacing [2,3]. The last equation is valid at $T \ll \hbar \omega / k_{B}$. The rate is expressed explicitly in $R$ to emphasize that $W_{n-1, n}$ depends exponentially on $T$ but only linearly on $R$. Thus, a very small overestimate of $T$ could result in a huge increase in the extracted value of $R$. For this reason, in order to obtain $R$ from the fit unambiguously, it is necessary to have $T$ independently verified.

It is well known that an effective system temperature significantly higher than the bath temperature $T_{b}$ often indicates serious problems in shielding the sample from
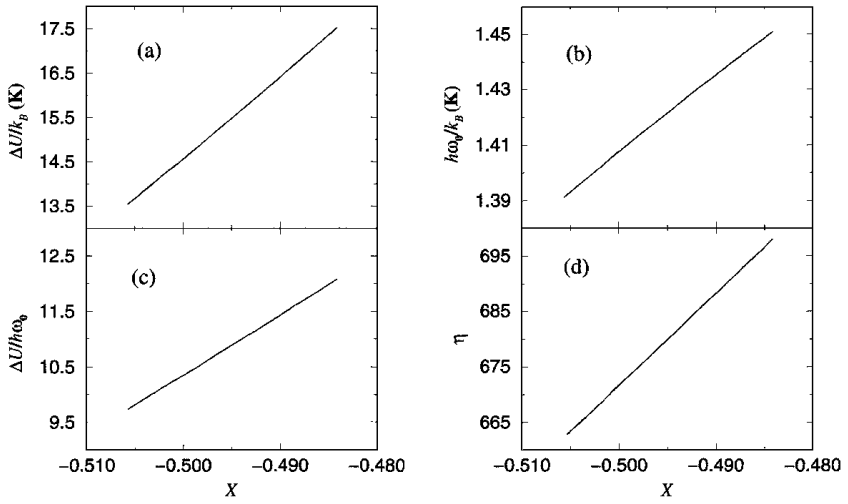

FIG. 1. Several key system parameters vs the normalized external flux calculated using SQUID parameters given in Ref. [1]. Note, the number of levels in the well is $N \approx$ $\operatorname{round}\left(\Delta U / \hbar \omega_{0}+0.5\right)$.

extrinsic electromagnetic noise. Therefore, treating $T$ as an adjustable fitting parameter, especially in a range well above $T_{b}$, requires justification. The fact that the system was observed to follow Kramers' thermal activation behavior down to $T_{b} \approx 1 \mathrm{~K}$ [1] strongly suggests that the effective system temperature was, in fact, much less than $0.5 \mathrm{~K}$, especially at $T_{b}<50 \mathrm{mK}$.

In summary, in Ref. [1] the energy level structure seems to have been miscalculated, which would entirely invalidate the escape rate calculations. More importantly, the rate of repopulating the upper level depends exponentially on $T$ so that the escape rate of a system with low damping resistance at low temperature mimics that of a system with a slightly higher temperature and a much larger $R$. Since the experimental evidence strongly indicates $T<0.5 \mathrm{~K}$, we conclude that the authors have significantly overestimated the effective damping resistance due to their inadequate data analysis procedure.

We thank Dr. D. J. Flees for critical readings of the manuscript and helpful discussions.

Siyuan Han

Department of Physics and Astronomy,

University of Kansas, Lawrence, Kansas 66045

R. Rouse

Sun Microsystems, Sunnyvale, California 94086

Received 2 August 1999; revised manuscript received 22 September 2000

DOI: 10.1103/PhysRevLett.86.4191

PACS numbers: 74.50.+r, 03.65.-w, 85.25.Dq

[1] C. Cosmelli, P. Carelli, M. G. Casteliano, F. Chiarelio, G. D. Palazzi, R. Leoni, and G. Torrioli, Phys. Rev. Lett. 82, 5357 (1999).

[2] W. Bialek, S. Chakravarty, and S. Kivelson, Phys. Rev. B 35, 120 (1987).

[3] A. N. Cleland, J. M. Martinis, and J. Clarke, Phys. Rev. B 37, 5950 (1988). 